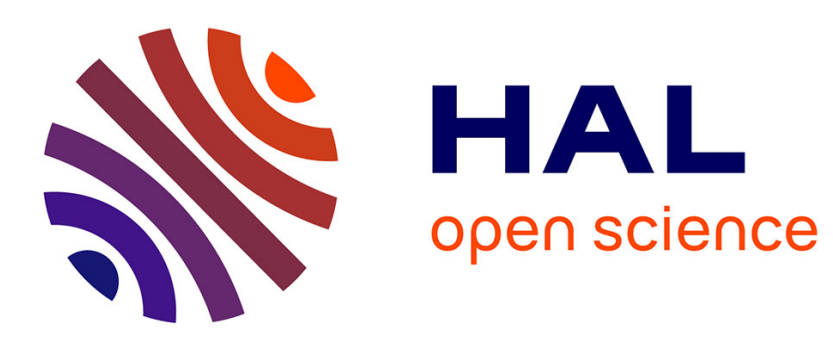

\title{
Quantifying resonance through a Lewis Valence Bond approach: application to haloallyl and carbonylcations
}

\author{
Mathieu Linares, Stéphane Humbel, Benoît Braïda
}

\section{To cite this version:}

Mathieu Linares, Stéphane Humbel, Benoît Braïda. Quantifying resonance through a Lewis Valence Bond approach: application to haloallyl and carbonylcations. Faraday Discussions, 2007, 135, pp.273283. 10.1039/B605325D . hal-01627885

\section{HAL Id: hal-01627885 https://hal.science/hal-01627885}

Submitted on 10 Nov 2017

HAL is a multi-disciplinary open access archive for the deposit and dissemination of scientific research documents, whether they are published or not. The documents may come from teaching and research institutions in France or abroad, or from public or private research centers.
L'archive ouverte pluridisciplinaire HAL, est destinée au dépôt et à la diffusion de documents scientifiques de niveau recherche, publiés ou non, émanant des établissements d'enseignement et de recherche français ou étrangers, des laboratoires publics ou privés. 


\title{
Quantifying resonance through a Lewis Valence Bond approach: application to haloallyl and carbonyl cations
}

\author{
Mathieu Linares, ${ }^{a}$ Stéphane Humbel ${ }^{* a}$ and Benoît Braïda ${ }^{* b}$
}

A recent Valence Bond scheme based on Lewis structures, the VBB method (M. Linares, B. Braida and S. Humbel, J. Phys. Chem. A, 2006, 110, 2505$2509)^{1}$ is applied to resonance effect quantification. An accurate evaluation of this effect is provided by targeting $\pi$ interactions only, while all other factors remain constant. Valence Bond theory allows us to circumvent two main shortcomings of other approaches, i.e. the lack of a quantitative aspect, and the difficulty to properly separate resonance from other effects. The $\pi$ effect of fluorine and chlorine atoms is found to be comparable and quite significant $\left(\sim 20 \mathrm{kcal} \mathrm{mol}^{-1}\right)$, in both haloallyl and protonated carbonyl cations. The validity of the resonance model for carbonyl compounds is confirmed. Resonance in protonated formamide is indeed found to be significantly larger than in formic acids, itself being more resonant than the formyl fluoride cation. Comparisons with other methods of resonance effect evaluation are also made.

\section{Introduction}

Substituent effect is one of the key concepts in chemistry. It is related to how an individual part of a molecule influences its general properties, and is usually separated into inductive, resonance and steric contributions. The inductive effect is the change in bond polarization triggered by the electronegativity differences between the substituent and the substrate. Resonance, or $\pi$-effect, corresponds to the delocalization of a substituent $\pi$ orbital (in general a $\pi$ lone pair) over the whole molecule. As the inductive effect corresponds to the change in polarization of already existing bonds, the resonance effect may be seen as a creation of a new and partial $\pi$ bond between substituent and substrate. The two effects are somehow connected, because the creation of a $\pi$ interaction (resonance effect) usually leads to a change in polarization of all bonds, that is to say a change in the inductive effect.

Many explanations in fundamental organic chemistry make use of substituent effects to account for different reactivity between different molecular analogues. Chemists have thus long devised models to compare relative electronic effects of different substituents. ${ }^{2}$ Hammett first defined a substituent constant, through a

\footnotetext{
${ }^{a}$ Université Paul Cézanne (Aix-Marseille III)/CNRS, UMR 6180 Chirotechnologies: Catalyse et Biocatalyse, Campus St Jérôme Case A 62, 13397 Marseille Cedex 20, France. E-mail: stephane.humbel@univ-cezanne.fr; mathieulinares@hotmail.com

${ }^{b}$ Université Pierre et Marie Curie-Paris 6/CNRS, UMR 7616-Laboratoire de Chimie Théorique, "Le Raphaël”, 3 rue Galilée, 94200 Ivry-Sur-Seine Cedex, France. E-mail: braida@lct.jussieu.fr
} 
general relation involving reaction rates or equilibrium constants for substituted and unsubstituted compounds, respectively. ${ }^{2,3}$ Through this equation and the numerous refinements that followed, ${ }^{4}$ the effects of individual substituents have been estimated for various chemical reactions and are expressed by different scales. These constants remain more or less proportional, and can be transferred from one reaction to another, providing an appreciable predictive tool for chemists. In particular, one kind of Hammett-derived equation was first proposed by Taft, ${ }^{5}$ which explicitly separates the inductive and resonance contributions. Despite their considerable use, these kinds of approaches suffer from several restrictions. Firstly, all of them are empirical, lacking a firm theoretical basis. Secondly, they are loaded with experimental errors, often of unknown magnitude. Besides, in order to separate inductive and resonance contributions, a reference system with no mesomeric contribution is postulated, which is never exactly the case. More importantly, these constants, though connected with observable quantities, do not directly correspond to the physically meaningful value, i.e. the energetic stabilization or destabilization induced by the effect at work.

These last few years, studies making use of quantum chemistry for evaluating these effects have become widespread in the literature. Theoretical methods are indeed able to provide several alternative ways of assessing inductive and resonance contributions. Some studies make use of population analysis for separating these effects. $^{6-8}$ All the arguments invoked are somewhat indirect, since the different methodologies rely on quantities that are not observables and depend on an approximate model. Moreover, these models give as well some indicators but not directly the relevant quantitative measure of the resonance effect, i.e. the energetic stabilization due to substituent $\pi$-orbital delocalization. Jug et al. have proposed a partition of the total electronic energy of a molecule into $\sigma$ and $\pi$ components. They have applied their approach on monosubstituted benzene by looking at the perturbations on the energies induced by different substituents, hence giving indications on differential resonance effects. ${ }^{9-11}$ However, the most popular way of assessing a substituent $\pi$-effect is still through evaluation of rotation barriers. It has been applied in particular for the evaluation of amide resonance, which has led to many interesting contributions. ${ }^{12-16}$ However, the rotated side-group still has with the substrate some resonance interaction through hyperconjugation, and this methodology usually underestimates the $\pi$-effect. ${ }^{17,18}$ Another important limitation inherent to all these approaches, is their ability to properly separate resonance from the polarization (inductive) contribution as a close connection exists between these two effects.

A better understanding of the substituent effect on reactivity would hence require a tool that combines three features, first an interpretation based on chemists' language, second a clean separation of the different effects at work, and third an accurate quantification through an energetic term. Such requirements can be fulfilled, at least for $\pi$-effects, in the framework of valence bond (VB) theory, which has often proved to provide additional insight relative to MO theory by expressing wavefunctions in a "chemical" point of view. We have used here a newly devised Lewis Valence Bond type of approach, the Valence Bond BOND (VBB) method. ${ }^{1}$ This method combines an extremely compact VB wavefunction, with a direct readability in terms of Lewis structures. We have used this method to measure in situ $\pi$-effects, through a direct measure of the energetic stabilization via substituent $\pi$-electrons delocalization, all other factors remaining constant. This can be carried out in the VBB framework with a minimal expansion of the Valence Bond wavefunction, always keeping a form where each component directly corresponds to a specific "chemical" Lewis structure. In addition, the VBB method can take into account a relevant part of the differential electron correlation, thus providing a rigorous and accurate estimation of energy stabilization due to resonance effects, as compared with the most accurate BOVB Valence Bond method. ${ }^{19,20}$ The methodology is first introduced through $\pi$-effect quantification of fluorine and chlorine in 
haloallyl cations. Different carbonyl systems will then be considered, resonance often being invoked to explain their contrasted reactivity under nucleophilic additions.

\section{Results and discussion}

\section{$\pi$-Effects on haloallyl cations}

The allyl cation was chosen as a test case for defining the VBB method, as it is a simple and highly resonating system. Fluoro- and choro-allyl cations hence appeared as natural first choices for the present study. Interestingly enough, halides are sometimes said to have negligible or no $\pi$ effect, ${ }^{21,22}$ whereas in chemistry textbooks this effect is sometimes found in mechanistic explanations, such as electrophilic attack on halobenzene. ${ }^{23}$ Haloallyl cations can be considered indeed as models of intermediate systems in addition reactions on halogen-substituted aromatic compounds. Besides, as fluorinated and chlorinated analogues demonstrate a different reactivity, it would be of interest to compare their respective $\pi$-effect.

Geometries of the two molecules as optimized at the MP2/cc-pvdz level of calculation are displayed in Fig. 1, along with the most relevant geometrical parameters. Both molecules are planar. The Lewis description of the haloallyl cation is pictured in Scheme 1. In this representation, the lone pairs of the halogen, and particularly the $\pi$ lone pair, are considered to remain strictly localized on the halogen atom, precluding any resonance interaction with the allyl moiety. The dashed bond in structure III represents a through space $\pi$ bond between the two atoms. Such a long-bond structure may be considered as exotic at first sight, but it has proved necessary in order to reach a realistic value for the allyl cation resonance., ${ }^{1,24}$

The VBB wavefunction attached to Scheme 1 above contains three components (VBB structures), each corresponding exactly to one of the Lewis structures. At this stage, it is important to recall the exact physical meaning of structures used here, as compared with traditional definitions of Valence Bond theory. In the Pauling Valence Bond definition of the wavefunction, each "active" bond in a Lewis structure ( $\pi$ bonds in this case) would be expanded in its covalent and ionic contributions. In Scheme 1, however, each $\pi$-bond should be understood as an implicit mixing of covalent and ionic situations. Such an interpretation of the chemical bond symbol is thus in line with modern conception. Even the simplest homonuclear bond like $\mathrm{H}_{2}$ is indeed better described as a superposition of covalent and ionic forms, rather than by the original purely covalent model. ${ }^{25,26}$ As a result, the traditional Pauling Valence Bond definition of the allyl cation wavefunction



Fig. 1 Geometries of fluoroallyl (top) and chloroallyl (bottom) cations. Bond lengths are in $\AA$, angles in ${ }^{\circ}$. 


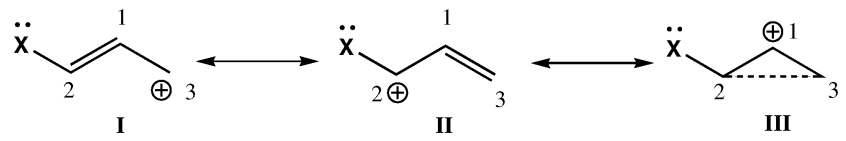

Scheme 1 Resonance model for haloallyl cations using Lewis structures (VBB description), with halogen $\pi$-effect switched off.

requires six Valence Bond structures, instead of three Lewis structures for the VBB wavefunction. A description corresponding to Scheme 1 can be achieved through the VBB method in the following way. First, the $\sigma$ orbitals are set as "inactive" orbitals. In the first level of VBB calculation (VBB-HF scheme), they are common to the three Lewis structures, and consist in Hartree-Fock MOs. The $\pi$ system is defined as the "active" set of orbitals and corresponding electrons, and is described in the following manner. Each $\pi$ bond consists of a pair of singlet-coupled Coulson-Fisher type of orbitals, ${ }^{25}$ delocalized between the two bonded atoms, so as to implicitly include both covalent and ionic terms within each pair bond. This part of the definition is similar to the bond distorted orbital approach. ${ }^{27}$ To reach a consistent description for all active electrons, the $\pi$ lone pairs of Scheme 1 is well described using a set of two singlet-coupled orbitals, both remaining, however, strictly localized on the halogen atom. Last, whereas all three structures bear common $\sigma$ orbitals at the VBB-HF level, each one has its own and specific set of active orbitals at the VBBbopt level, thus having different $\pi$ orbitals for different structures in the spirit of the BOVB method from Hiberty et al. ${ }^{20,28}$ All $\pi$ orbitals and coefficients of VBB structures are optimized simultaneously, following the variational principle, so as to reach the lowest energy solution. The VBB definition of the wavefunction was found in our previous study to include non-dynamic (in particular "left-right") correlation, as well as a relevant part of differential dynamic correlation.

The description of the haloallyl cation in Scheme 1 does not allow any resonance interaction from the halogen $\pi$ lone pair, as the latter remains strictly localized on the halogen atom. The VBB wavefunction attached to Scheme 1 will thus be called $\Psi_{\text {loc }}$. It is worth noting that in such a wavefunction, whereas the $\pi$-effect is switched off, the complete inductive effect is built in the wavefunction and corresponds to the ground state situation, through the Hartree-Fock $\sigma$ frame that is optimized in the delocalized ground state. Allowing $\pi$ lone pair delocalization would correspond to the representation of the haloallyl cation presented in Scheme 2. Only the second structure changes between the two descriptions, a partial $\pi$ bond between the halogen and its connected carbon atom being for now on created through delocalization (structure $\mathrm{II}^{\prime}$ ). Structure $\mathrm{II}^{\prime}$ is included in the computation by simply letting the two orbitals of the halogen lone pair delocalize onto the neighbouring $\mathrm{C} 2$ atom. As a result, this second definition of the VBB wavefunction for haloallyl cations now takes into account the halogen resonance effect through structure $\mathrm{II}^{\prime}$, and will be referred to as $\Psi_{\text {deloc }}$. The energy difference between the delocalized ground state $\Psi_{\text {deloc }}$ and the localized diabatic state $\Psi_{\text {loc }}$, the Vertical Delocalization Energy (VDE), corresponds precisely to the energetic quantification of halogen resonance effect. The term "Vertical" means that localized and delocalized states both have the same geometry. Hence, switching on or off the delocalization is peformed in a simple way, and without any extra expansion of the VBB wavefunctions for haloallyl



Scheme 2 Resonance model for haloallyl cations using Lewis structures, with halogen $\pi$-effect switched-on. 
Table 1 Hartree-Fock and Valence Bond total energies (hartree), along with Vertical Delocalization Energies $\left(\mathrm{kcal} \mathrm{mol}^{-1}\right.$ ), for fluoroallyl and chloroallyl cations

\begin{tabular}{|c|c|c|c|c|c|}
\hline & & \multicolumn{2}{|l|}{$\mathrm{X}=\mathrm{F}$} & \multicolumn{2}{|l|}{$\mathrm{X}=\mathrm{Cl}$} \\
\hline & & $\bar{E}$ & $\overline{\mathrm{VDE}}$ & $\bar{E}$ & VDE \\
\hline \multirow{3}{*}{$\begin{array}{l}\text { HF } \\
\text { VBB-HF }\end{array}$} & & -215.059772 & - & -575.124172 & - \\
\hline & $\Psi_{\mathrm{loc}}$ & -215.056682 & 0.0 & -575.119418 & 0.0 \\
\hline & $\Psi_{\text {deloc }}$ & -215.094349 & -23.6 & -575.152479 & -20.7 \\
\hline \multirow[t]{2}{*}{ VBB-bopt } & $\Psi_{\mathrm{loc}}$ & -215.087828 & 0.0 & -575.150862 & 0.0 \\
\hline & $\Psi_{\text {deloc }}$ & -215.116762 & -18.2 & -575.173730 & -14.3 \\
\hline
\end{tabular}

cations. This has to be compared with traditional Pauling Valence Bond treatment, where it would imply adding six extra Valence Bond structures (going from 6 VB structures for the localized case, to $12 \mathrm{VB}$ structures for the delocalized case). The VBB scheme hence combines greater compactness, with a direct readability in terms of Lewis structures. Most importantly, the VBB method takes advantage of Valence Bond flexibility in allowing a clear separation of resonance from other kinds of effects (inductive, steric, etc.). Only the substituent-substrate $\pi$ interaction is modified, all the remaining factors ( $\sigma$ electronic density, geometry) remaining fixed to the ground state situation.

The total energies of the different wavefunctions, as well as the VDE for fluoroallyl and chloroallyl cations are displayed in Table 1. The Hartree-Fock energy is given as well for the sake of comparison. The VBB-HF values, corresponding precisely to the methodology just presented, will be considered first. The VDE of both fluorine and chlorine appears far from being negligible in haloallyl cation, up to 23.6 and $20.7 \mathrm{kcal} \mathrm{mol}^{-1}$, respectively. This is more than one third of the resonance energy in allyl cation itself, $54 \mathrm{kcal} \mathrm{mol}^{-1}$ at the same level of computation. This result is in sharp contrast with the allegedly negligible resonance halogen effect reported by Wiberg et al. ${ }^{22}$ It most likely originates from the great mobility of $\pi$ electrons in the positively charged allyl fragment, and it would certainly be less important in a neutral system. As a result, the magnitude of this effect justifies its invocation in reaction mechanisms of positively charged resonant systems, such as electrophilic additions on halobenzenes. Another important revelation is the surprising closeness between the VDEs for fluorine and chlorine, with only $3.1 \mathrm{kcal}$ $\mathrm{mol}^{-1}$ of difference in favor of the former atom. The $\pi$ effect of the two atoms is found to be very similar, so it cannot explain the contrasted reactivity of chloro- $v s$ fluoro-substituted molecules. The root cause must lie in other effects, possibly in a difference in inductive effect.

Weights of individual structures is a fundamental parameter which naturally come out of a Valence Bond calculation. The VBB method provides weights associated to the Lewis structures depicted in Scheme 1 (for $\Psi_{\text {loc }}$ ) or in Scheme 2 (for $\Psi_{\text {deloc }}$ ).

Table 2 VBB-HF weights ${ }^{a}$ of Lewis structures composing localized and delocalized wavefunctions, for fluoroallyl and chloroallyl cations

\begin{tabular}{llllll}
\hline & $\mathrm{X}=\mathrm{F}$ & & $\mathrm{X}=\mathrm{Cl}$ & \\
\cline { 2 - 3 } \cline { 5 - 6 } & $\Psi_{\text {loc }}$ & $\Psi_{\text {deloc }}$ & & $\Psi_{\text {loc }}$ & $\Psi_{\text {deloc }}$ \\
\hline I & 30 & 22 & 36 & 21 \\
II or II & 48 & 69 & & 39 & 63 \\
III & 22 & 09 & 25 & 16 \\
${ }^{a}$ Coulson-Chirgwin weights. ${ }^{29}$ & & & \\
\hline
\end{tabular}


Values are presented in Table 2, following the Coulson-Chirgwin definition. ${ }^{29}$ As expected, III is a minor structure in both cases. Quite interestingly, structure II has systematically a larger weight than structure $\mathrm{I}$, which is inconsistent with a larger $\mathrm{C}_{1}-$ $\mathrm{C}_{2}$ bond distance as compared with $\mathrm{C}_{1}-\mathrm{C}_{3}$. When the halogen $\pi$-effect is switched on, the weights of structures I and III logically go down, and structure II' becomes largely dominant. Structure II' has a slightly larger weight in fluoroallyl than in chloroallyl cations, consistent with a slightly larger VDE.

In our previous study on allyl cation resonance, we found that using common Hartree-Fock MOs for all resonating structures (VBB-HF level) led to an underestimation of the resonance energy. It is therefore of interest to go beyond the VBBHF level in this study as well. A second level of correlation (VBB-bopt in Table 1) is obtained by allowing each Lewis structure of the VBB wavefunction to have its specific set of $\sigma$ orbitals, different from one structure to another, in addition to their individual set of $\pi$ orbitals. All orbitals ( $\sigma$ and $\pi$ ) together with the coefficient of the structures are optimized simultaneously, giving the VBB-bopt wavefunction. This optimization of an individual $\sigma$ frame for each Lewis structure has been shown to introduce extra dynamic correlation in the wavefunction. The physical ingredient corresponds to the dynamic interplay between $\sigma$ and $\pi$ electrons, that is to say the instantaneous repolarization of the $\sigma$ electronic density induced by the dynamic $\pi$ electrons move from one bonding situation to another. When going from the VBBHF to the VBB-bopt level, the total energy logically diminishes as more correlation is introduced. This energy lowering is superior in the diabatic states than in the ground state, because the Hartree-Fock $\sigma$ MOs are adapted to the delocalized situation. As a result, the VDE is lower with VBB-bopt compared with VBB-HF, by 5.4 and $6.4 \mathrm{kcal} \mathrm{mol}^{-1}$ for fluoroallyl and chloroallyl, respectively. Which level is the most relevant for our current purpose, is rather a matter of choice. This extra $\sigma-\pi$ correlation energy is nothing more than the energetic consequence of the variation in inductive effect when going from one resonating situation to another. Hence, the VBB-bopt VDE can be seen to include part of the inductive effect, precisely the change in inductive effect caused by the creation (or suppression) of the $\pi$ effect. On the other hand, at the VBB-HF level the $\sigma$ set of orbitals is common to both states and optimized for the delocalized situation, so this method gives an in situ measure of the $\pi$ effect in the presence of an intact $\sigma$ frame. For a better understanding of the different effects at work in chemical reactions, a clear separation between them is needed, the VBB-HF level is preferred and considered in the following. However, whatever choice is made, it should be noticed that the differential resonance effect between fluorine and chlorine remains similar with both levels of computation.

\section{$\pi$-Effects on carbonyl groups}

Carbonyl is one of the most fundamental functional groups in organic chemistry. This family of molecules is often divided into divalent (aldehydes and ketones) and trivalent functional groups (carboxylic acids and derivatives). However ubiquitous these functions may be, open questions still remain on some of their fundamental properties. Among them, whether electrostatic or resonance effect is the root cause

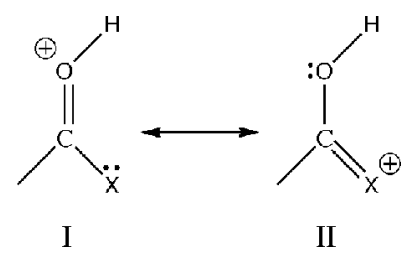

Scheme 3 Resonance model in protonated carbonyl groups, using Lewis structures (VBB description). 



Fig. 2 Geometries of second row (first line) and third row (second line) substituted carbonyl cations. Bond lengths are in $\AA$, angles in ${ }^{\circ}$.

for the particular acidity of carboxyl compounds, ${ }^{30-33}$ as well as the factors at the origin of barrier rotation in amides and derivatives ${ }^{12,13,16,17,34}$ have been the subject of heated debates. Another matter concerns the usual explanation for the contrasted reactivity of different carbonyl based functional groups. In particular, formyl chlorides are known to be very reactive under a nucleophilic attack, whereas amides are much less so, with acids and esters lying in between. This contrasted reactivity of carboxylic derivatives is traditionally explained in chemistry textbooks through a stabilizing resonance interaction in the reactant. ${ }^{35}$ This resonance effect being absent in the tetravalent pyramidal transition state, the reaction barriers would be larger if there were a strong resonance in the reactant molecule. This resonance stabilization is shown in Scheme 3 for the acid-catalyzed reagent, where a proton is attached to the oxygen of the carbonyl after the initiation step.

Although this explanation is well accepted among chemists, to our knowledge no accurate $\pi$ effect quantification in terms of the resonance energy has been given for these cations, in connection with their well-known differences in reactivity. This prompted us to apply our methodology on a representative range of protonated carbonyl compounds, in order to quantify and compare the resonance effect due to different substituents on the carbonyl groups. Limiting ourselves to the second and third rows of the periodic table, the ensemble of mono-substituted carbonyl compounds is covered by the series: $\mathrm{HXCOH}^{+}$, with: $\mathrm{X}=\left(\mathrm{CH}_{3}, \mathrm{NH}_{2}, \mathrm{OH}, \mathrm{F}\right.$; $\left.\mathrm{SiH}_{3}, \mathrm{PH}_{2}, \mathrm{SH}, \mathrm{Cl}\right)$. This series includes in particular an example of a protonated carboxylic acid, an amide, as well as formyl fluoride and chloride. The geometries, as optimized at the MP2/cc-pvdz level of theory, are displayed in Fig. 2. In particular, the $\mathrm{C}-\mathrm{X}$ and $\mathrm{C}-\mathrm{O}$ bond lengths are shown. All molecules are of $C_{\mathrm{s}}$ symmetry.

In these molecules, as is the case in haloallyl systems, the resonance effect can be turned off and on at will, by respectively preventing or allowing the substituent $\pi$ orbital to delocalize on the carbonyl carbon atom. Switching off the delocalization would correspond to a VBB wavefunction ( $\left.\Psi_{\text {loc }}\right)$ composed only of one Lewis structure, i.e. structure I of Scheme 3, whereas turning on the delocalization would correspond to a VBB wavefunction $\left(\Psi_{\text {deloc }}\right)$ containing the two structures in resonance as depicted in Scheme $3 . \dagger$

$\uparrow$ A description in the framework of the traditional Pauling Valence Bond ansatz would require at least three Valence Bond structures for the delocalized ground state and two VB structures for the localized diabatic state. ${ }^{16,17}$ 
Table 3 VBB-HF Vertical Delocalization Energies (VDE) in kcal mol ${ }^{-1}$ along with sone $\sigma_{\mathrm{R}}{ }^{+}$ resonance constants ${ }^{a}$, for second and third row susbtituted carbonyl cations

\begin{tabular}{lcccc}
\hline & $-\mathrm{CH}_{3}$ & $-\mathrm{NH}_{2}$ & $-\mathrm{OH}$ & $-\mathrm{F}$ \\
\hline $\mathrm{VDE}$ & 09.5 & 58.3 & 36.0 & 22.0 \\
$\sigma_{\mathrm{R}}{ }^{+}$ & -0.08 & -0.52 & -0.38 & -0.25 \\
\hline & $-\mathrm{SiH}_{3}$ & $-\mathrm{PH}_{2}$ & $-\mathrm{SH}$ & $-\mathrm{Cl}$ \\
\hline $\mathrm{VDE}$ & 03.0 & 57.0 & 33.5 & 21.0 \\
$\sigma_{\mathrm{R}}{ }^{+}$ & & & -0.25 & -0.17 \\
${ }^{a}$ Values taken from ref. 4. & & & \\
\hline
\end{tabular}

The energy differences between $\Psi_{\text {deloc }}$ and $\Psi_{\text {loc }}$ wavefunctions (VDE) are shown in Table 3. We shall first look at the trivalent functional groups of the second row $(\mathrm{X}=$ $\mathrm{NH}_{2}, \mathrm{OH}, \mathrm{F}$ ). With a VDE of $22.0 \mathrm{kcal} \mathrm{mol}^{-1}$, the $\pi$-effect in protonated formyl fluoride appears to be very similar to that found for the fluoroallyl cation. As expected, this $\pi$-effect increases when going to the less electronegative $-\mathrm{OH}$ substituent, with a VDE of $36.0 \mathrm{kcal} \mathrm{mol}^{-1}$. It goes up to the quite impressive value of $58.3 \mathrm{kcal} \mathrm{mol}^{-1}$ for protonated formylamide cation, the $\pi$-effect being almost three times larger in this amide derivative than in the protonated formyl fluoride. This difference in resonance effect between different functional derivatives is large enough to be considered as being at least one of the root causes for their well-known differences in reactivity under nucleophilic attack. This validates the traditional textbook explanation, as depicted in Scheme 3. Let us now consider the third row carboxylic derivatives $\left(\mathrm{X}=\mathrm{PH}_{2}, \mathrm{SH}, \mathrm{Cl}\right.$ ). Quite in line with that found for haloallyl cations, the $\pi$-effect appears to be very similar for third row substituents, though systematically slightly lower. The difference in VDE ranges from $1 \mathrm{kcal} \mathrm{mol}^{-1}$ between fluorine and chlorine, to $2.5 \mathrm{kcal} \mathrm{mol}^{-1}$ between acid and thioacid, with amide and phospho-amide being somewhere in between with a difference of $1.3 \mathrm{kcal}$ $\mathrm{mol}^{-1}$. As a result, the $\pi$ delocalization ability appears to be similar for corresponding second and third row substituents. The difference in reactivity between carbonyl analogues comes from other factors, presumably from differences in inductives effects. $^{33}$

The last group of substituents, i.e. methyl and silyl group, should be taken aside. They can show a resonance effect on carbonyl groups, however, not through a pure $\mathrm{p}$ lone pair as in trivalent functions, but by hyperconjugation interaction, i.e. delocalization of electrons occupying a bonding $\mathrm{C}-\mathrm{H}$ or $\mathrm{Si}-\mathrm{H}$ fragment orbital of $\pi$ symmetry. Whatever the origin of the $\pi$ electrons in substituents, both situations (pure lone pair or $\pi$ bonding orbital) can be treated on the same footing with the VBB method. The $\pi$-effect on carbonyl is expected to be significantly lower than traditional conjugation coming from a p lone pair, and this is confirmed by the much smaller, though not negligible, VDE for these divalent species, respectively, $9.5 \mathrm{kcal}$ $\mathrm{mol}^{-1}$ for methyl and only $3.0 \mathrm{kcal} \mathrm{mol}^{-1}$ for silyl.

In the beginning of this paper, we have mentioned the widely used Hammett-Taft constants for assessing inductive $\left(\sigma_{\mathrm{I}}\right)$ and resonance $\left(\sigma_{\mathrm{R}}\right)$ effects of substituents. In the second part of Table 3 , we show some resonance constant values taken from the literature. The correlation with our computed VDE appears to be surprisingly good for second row substituted carbonyls, considering all the uncertainty underlying Hammett constants as well as the supposed lesser transferability of resonance as compared to inductive constants. This may imply that the general trend found on protonated carbonyl could be extended to other systems with highly delocalized $\pi$ electrons, like substituted benzene cations for instance. This hypothesis is supported by the extreme closeness between VDEs on haloallyl cations $v s$ corresponding protonated formyl halides. However, this correlation does not seem to extend to 
Table 4 VBB-HF along with NRT weights ${ }^{a}$ for structure II' (in \%)

\begin{tabular}{lllll}
\hline & $-\mathrm{CH}_{3}$ & $-\mathrm{NH}_{2}$ & $-\mathrm{OH}$ & $-\mathrm{F}$ \\
\hline VBB-HF & 32 & 58 & 49 & 42 \\
NRT & 14 & 62 & 48 & 36 \\
\hline & $-\mathrm{SiH}_{3}$ & $-\mathrm{PH}_{2}$ & $-\mathrm{SH}$ & $-\mathrm{Cl}$ \\
\hline VBB-HF & 32 & 52 & 56 & 47 \\
NRT & 08 & 68 & 57 & 43 \\
${ }^{a}$ Coulson-Chirgin weights. & ${ }^{28}$ & & & \\
\hline
\end{tabular}

third row $v s$ second row resonance effect comparisons. The few $\sigma_{\mathrm{R}}{ }^{+}$values displayed in Table 3 for third row molecules indeed appears to be quite different from the corresponding second row values, in contradiction to that found with VDEs. A significant difference between resonance constants of second and third row substituents was also found using another theoretical model. ${ }^{6}$ We believe that these empirical methods include a mixture of other electronic effects (presumably inductive) inside their resonance indicators, to an amount differing in magnitude on going down the periodic table. This unbalanced mixing would then spoil the comparison between substituents of different rows.

The general trends on $\pi$-effects found through VDE should also be apparent in the composition of the VBB wavefunctions. Hence, a substituent with a strong $\pi$-effect should lead to a $\Psi_{\text {deloc }}$ wavefunction with a dominant structure II. This can be checked looking at Table 4, where the respective weights of structures I and II in Lewis-type wavefunctions are displayed. In addition to the weights that naturally arise from the VBB computations, we also show NRT weights calculated from an $a$ posteriori NBO analysis onto a B3LYP wavefunction. Indeed, the NRT method allows a delocalized MO-based electronic density to be projected into Lewis structures. ${ }^{36-38}$ Despite the differences in methodologies and correlation methods (Valence Bond vs DFT), the VBB and NRT weights appears to agree well with each other, except for methyl and silyl substituents that may be a limiting case for the $\mathrm{NBO} / \mathrm{NRT}$ techniques. $\ddagger$ Structure II is expectedly larger in wavefunction attached to the strongest $\pi$-donors. It should be mentioned here that the NRT analysis also allows retrieval of some resonance energies, however, resonance seems to be largely overestimated. ${ }^{39}$ Consequently, if one is interested in the weights of Lewis structures only, the NBO/NRT method applied on correlated electronic densities could be a cheap and convenient alternative to our Lewis Valence Bond computational scheme. However, if a precise quantification of $\pi$-effects through delocalization energies is required, the Valence Bond BOND scheme remains the method of choice.

\section{Computational details}

Our study used MP2/cc-pvdz geometries as optimized with the Gaussian package. ${ }^{40}$ The B3LYP and VB calculations were made with the same cc-pvdz ${ }^{41}$ basis set. For these calculations, we used the XMVB program from $\mathrm{Wu}$ and co-workers. ${ }^{42}$ The XMVB program is a modern and efficient spin-free Valence-Bond code, allowing full flexibility for the definition of the Valence-Bond wavefunction. Additional computations with the Natural Resonance Theory (NRT) were made using the NRT code embedded in the NBO 5.0 program. ${ }^{43}$

\$ With the NBO/NRT techniques, the methyl subsituent $\mathrm{C}-\mathrm{H}$ bonds involved in the $\pi$ donation should be broken to form a double bond between connected carbons. Thus structure II' cannot be directly introduced into an NRT analysis, but is described through several sub-structures. 


\section{Conclusion}

The Valence Bond BOND (VBB) method appears to be a convenient tool for cleanly separating resonance from other substituent effects, and for providing accurate quantification through vertical delocalization energies (VDE). This scheme makes proper use of the natural flexibility of Valence Bond methods, in allowing delocalization to be turned on and off, at will, in a conjugated molecule. The VBB wavefunctions are composed of Lewis structures, in opposition to traditional Pauling Valence Bond description (covalent and ionic expansion of the chemical bond). This allows a more compact description of resonating molecules to be used, as well as readability of the wavefunction in a more chemical sense.

This methodology is applied to resonance effect quantification in two families of substrates: the haloallyl and protonated carbonyl cations. The $\pi$ electrons were shown to be an important feature of the electronic structures of these functional groups, the substituent lone pairs being significantly delocalized over the substrate moiety. For fluorine and chlorine substituents, a significant and similar resonance effect of about $20-23 \mathrm{kcal} \mathrm{mol}^{-1}$ is found. This confirms previous signs of a significant halogen resonance effect in cations, whereas this effect was found to be negligible in neutral molecules. ${ }^{21}$ The $\pi$ effect appears to be almost identical in haloallyl and in carbonyl cations, thus pointing towards its transferability at least to other resonating cations.

Computations on protonated carbonyl derivatives have shown that these results put forward different pairs of corresponding second and third row substituents. Similarity in $\pi$ donor ability has indeed been found between $-\mathrm{OH}$ and $-\mathrm{SH},-\mathrm{NH}_{2}$ and $-\mathrm{PH}_{2}$, and to a lower extent $-\mathrm{CH}_{3}$ and $-\mathrm{SiH}_{3}$. It can be inferred that differences in reactivity between second and third row substituted molecules come from other effects, differences in inductives effects presumably playing a significant role.

This study supports as well the traditional resonance model in carboxylic compounds. The resonance effect is significantly larger in protonated carboxylic acid than in acyl fluoride, and is found to be maximal in protonated formamide. The differences are large enough to explain the contrasted reactivity of carboxylic compounds under nucleophilic attack, altough other effects may contribute as well.

Comparisons between VDEs and other resonance indicators are also made. Hammett-Taft constants give similar trends to our computed VDEs for second row substituents, however, they seem to fail in comparing the resonance effect between second and third row substituents. This was attributed to an imperfect separation of the $\pi$ effect combined with an ill-balanced mixing with other effects in these constants. Lastly, the weight of Lewis structures from VBB computations and from a NRT analysis onto B3LYP wavefunctions are found to be similar.

\section{Acknowledgements}

Pr Wei Wu is most gratefully acknowledged for providing the XMVB code. M. L. is thankful to the French ministry of research (fellowship). This work was supported by substantial computing facilities from "CRIHAN, Plan Interrégional du Bassin Parisien" (project 2001-003), and "Centre Régional de Compétence en Modélisation Moléculaire de Marseille".

\section{References}

1 M. Linares, B. Braida and S. Humbel, J. Phys. Chem. A, 2006, 110, 2505-2509.

2 T. M. Krygowski and B. T. Stepien, Chem. Rev., 2005, 105, 3482.

3 L. P. Hammett, Trans. Faraday Soc., 1938, 34, 0156-0164.

4 C. Hansch, A. Leo and R. W. Taft, Chem. Rev., 1991, 91, 165-195.

5 R. W. Taft and I. C. Lewis, J. Am. Chem. Soc., 1959, 81, 5343-5352.

6 E. Dumont and P. Chaquin, J. Mol. Struct. (THEOCHEM), 2004, 680, 99-106.

7 O. Exner, M. Ingr and P. Carsky, J. Mol. Struct. (THEOCHEM), 1997, 397, 231-238. 
8 S. Marriott and R. D. Topsom, J. Chem. Soc., Perkin Trans. 2, 1985, 1045-1047.

9 K. Jug and A. M. Koster, J. Am. Chem. Soc., 1990, 112, 6772-6777.

10 K. Jug and M. Matuschewski, Int. J. Quantum Chem., 1994, 49, 197-206.

11 K. Jug, M. Matuschewski and A. M. Koster, J. Phys. Org. Chem., 1993, 6, 645-650.

12 K. E. Laidig and L. M. Cameron, J. Am. Chem. Soc., 1996, 118, 1737-1742.

13 J. K. Pugh and A. Streitwieser, J. Org. Chem., 2001, 66, 1334-1338.

14 K. B. Wiberg, J. R. Cheeseman, J. W. Ochterski and M. J. Frisch, J. Am. Chem. Soc., 1995, 117, 6535-6543.

15 K. B. Wiberg, P. R. Rablen, D. J. Rush and T. A. Keith, J. Am. Chem. Soc., 1995, 117, 4261-4270.

16 D. Lauvergnat and P. C. Hiberty, J. Am. Chem. Soc., 1997, 119, 9478-9482.

17 Y. R. Mo, P. V. Schleyer, W. Wu, M. H. Lin, Q. Zhang and J. L. Gao, J. Phys. Chem. A, 2003, 107, 10011-10018.

18 Y. R. Mo, J. Org. Chem., 2004, 69, 5563.

19 P. C. Hiberty, S. Humbel, C. P. Byrman and J. H. Van Lenthe, J. Chem. Phys., 1994, 101, 5969-5976.

20 P. C. Hiberty and S. Shaik, Theor. Chem. Acc., 2002, 108, 255-272.

21 K. B. Wiberg, C. M. Hadad, P. R. Rablen and J. Cioslowski, J. Am. Chem. Soc., 1992, 114, 8644-8654.

22 K. B. Wiberg and P. R. Rablen, J. Org. Chem., 1998, 63, 3722-3730.

23 K. P. C. Vollhardt and N. E. Schore, Organic Chemistry, Structure and Function, 4th edn, W. H. Freeman and Co., NY, 2003.

24 Y. R. Mo, Z. Y. Lin, W. Wu and Q. N. Zhang, J. Phys. Chem., 1996, 100, 6469-6474.

25 C. A. Coulson and I. Fischer, Philos. Mag., 1949, 40, 386-393.

26 L. Pauling, J. Am. Chem. Soc., 1931, 53, 3225-3237.

27 W. A. Goddard and L. B. Harding, Annu. Rev. Phys. Chem., 1978, 29, 363-396.

28 P. C. Hiberty, J. P. Flament and E. Noizet, Chem. Phys. Lett., 1992, 189, 259-265.

29 B. H. Chirgwin and C. A. Coulson, Proc. R. Soc. London, Ser. A, 1950, 201, 196-209.

30 O. Exner and P. Carsky, J. Am. Chem. Soc., 2001, 123, 9564-9570.

31 P. C. Hiberty and C. P. Byrman, J. Am. Chem. Soc., 1995, 117, 9875.

32 P. R. Rablen and K. H. Bentrup, J. Am. Chem. Soc., 2003, 125, 2142-2147.

33 K. B. Wiberg, Acc. Chem. Res., 1999, 32, 922-929.

34 K. B. Wiberg and P. R. Rablen, J. Am. Chem. Soc., 1995, 117, 2201-2208.

35 F. A. Carey and R. J. Sundberg, Advanced Organic Chemistry, 4th edn, Springer Science and Business Media Inc., New York, NY, 2001.

36 E. D. Glendening, J. K. Badenhoop and F. Weinhold, J. Comput. Chem., 1998, 19, 628-646.

37 E. D. Glendening and F. Weinhold, J. Comput. Chem., 1998, 19, 593-609.

38 E. D. Glendening and F. Weinhold, J. Comput. Chem., 1998, 19, 610-627.

39 Y. R. Mo and S. D. Peyerimhoff, J. Chem. Phys., 1998, 109, 1687-1697.

40 M. J. Frisch, G. W. Trucks, H. B. Schlegel, G. E. Scuseria, M. A. Robb, J. R. Cheeseman, V. G. Zakrzewski, J. J. A. Montgomery, R. E. Stratmann, J. C. Burant, S. Dapprich, J. M. Millam, A. D. Daniels, K. N. Kudin, M. C. Strain, O. Farkas, J. Tomasi, V. Barone, M. Cossi, R. Cammi, B. Mennucci, C. Pomelli, C. Adamo, S. Clifford, J. Ochterski, G. A. Petersson, P. Y. Ayala, Q. Cui, K. Morokuma, D. K. Malick, A. D. Rabuck, K. Raghavachari, J. B. Foresman, J. Cioslowski, J. V. Ortiz, B. B. Stefanov, G. Liu, A. Liashenko, P. Piskorz, I. Komaromi, R. Gomperts, R. L. Martin, D. J. Fox, T. Keith, M. A. Al-Laham, C. Y. Peng, A. Nanayakkara, C. Gonzalez, M. Challacombe, P. M. W. Gill, B. Johnson, W. Chen, M. W. Wong, J. L. Andres, C. Gonzalez, M. Head-Gordon, E. S. Replogle and J. A. Pople, Gaussian 98 rev. A9, Gaussian Inc., Pittsburgh, PA, 1998.

41 T. H. Dunning, J. Chem. Phys., 1989, 90, 1007-1023.

42 L. Song, W. Wu, Y. Mo and Q. Zhang, Xiamen University, Xiamen 361005, China, 2003.

43 E. D. Glendening, J. K. Badenhoop, A. E. Reed, J. E. Carpenter, J. A. Bohmann, C. M. Morales and F. Weinhold, NBO 5.0, Theoretical Chemistry Institute, University of Wisconsin, Madison, WI, 2001, http://www.chem.wisc.edu/ nbo5. 